\title{
Origin of giant spin-lattice coupling and the suppression of ferroelectricity in $\mathrm{EuTiO}_{3}$ from first principles
}

\author{
Turan Birol and Craig J. Fennie \\ School of Applied \& Engineering Physics, Cornell University, Ithaca, NY 14853, USA
}

(Dated: September 25, 2018)

\begin{abstract}
We elucidate the microscopic mechanism that causes a suppression of ferroelectricity and an enhancement of octahedral rotations in $\mathrm{EuTiO}_{3}$ from first principles. We find that the hybridization of the rare earth $\mathrm{Eu} 4 \mathrm{f}$ states with the B-site Ti cation drives the system away from ferroelectricity. We also show that the magnetic order dependence of this hybridization is the dominant source of spin-phonon coupling in this material. Our results underline the importance of rare earth $\mathrm{f}$ electrons on the lattice dynamics and stability of these transition metal oxides.
\end{abstract}

\section{INTRODUCTION}

In the last ten years there has been an intense effort to discover new materials that display strong magnetoelectric coupling. Such materials could enable novel devices in which an electric-field control magnetism.1 [4 In this pursuit first-principles computational methods have played a key role by successfully predicting new material realizations 5 even when the underlying microscopic mechanisms have not always been clear!16 Elucidating these mechanisms is important for both fundamental understanding and also for guiding the search for new magnetoelectrics. One example is the perovskite $\mathrm{EuTiO}_{3}{ }_{17}^{17}$

Bulk $\mathrm{EuTiO}_{3}$, shown in Fig. 17, is a paraelectric antiferromagnet that displays a dielectric anomaly at the magnetic ordering temperature $\left(T_{N} \sim 5.3 \mathrm{~K}\right){ }^{1819} \mathrm{Much}$ more relevant to the possibility of magnetoelectric phase control were the pioneering experiments of Katsufuji and Takagi, which showed that $\mathrm{EuTiO}_{3}$ exhibits a magnetodielectric effect; at low temperatures, the dielectric constant depends strongly on the magnitude of the external magnetic field ${ }^{19}$ They suggested that this effect stemmed from spin-phonon coupling, 20121 i.e., the dependence of the polar phonon frequencies on spin correlations. Subsequent first-principles studies ${ }^{22|23|}$ and direct measurements of the phonon frequencies under magnetic field $\underline{24 \mid 25}$ have largely confirmed this picture.

Regarding the magnetoelectric properties of $\mathrm{EuTiO}_{3}$, it was shown from first principles how the underlying physics leading to the observation of spin-phonon coupling can be exploited to enable control over the dielectric and the magnetic ground state. ${ }^{22}$ Epitaxial strain was proposed as the explicit control "knob" that tunes the antiferromagnetic-paraelectric ground state into a simultaneous ferromagnetic-ferroelectric phase. Furthermore it was argued that under increasing strain but before reaching this novel multiferroic phase, a giant magnetoelectric response would occur in the vicinity of the strain-induced phase transitions due to phase competition. 26/27] Subsequent experiments on epitaxially strained thin films have observed the strain induced ferromagnetic-ferroelectric phase ${ }^{28}$ and also the suppression of the antiferromagnetic order by an external electric

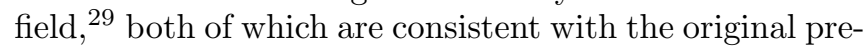
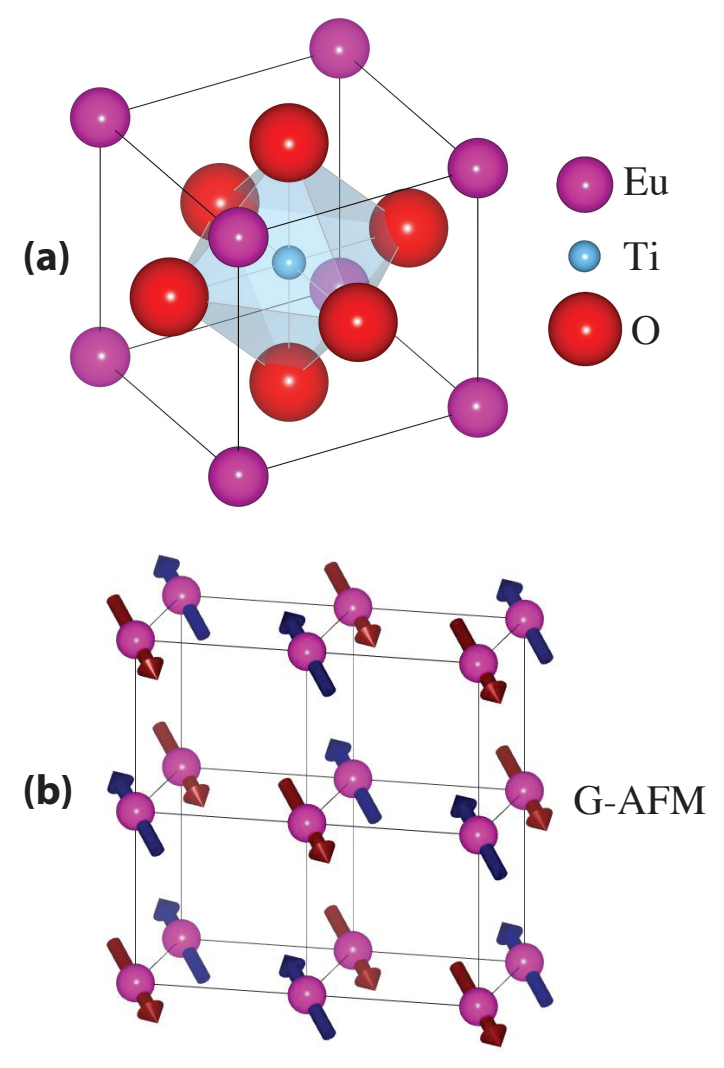

FIG. 1. (a) Crystal structure of perovskite $\mathrm{EuTiO}_{3}$ in the cubic phase. (b) Sketch of G-type antiferromagnetic order. Arrows denote the direction of spins.

diction, yet the giant magnetoelectric effect has yet to be observed (possibly due to a lack of high quality substrates that would provide the necessary value of strain).

While it has been suggested that the physics of $\mathrm{EuTiO}_{3}$ largely originates from a cation-mediated exchange mechanism, 1130 the microscopic mechanism of the spin-phonon coupling, and subsequently of the magnetoelectric phase control, is unknown. Here we ask an important, but overlooked, question whose answer makes these clear; Why isn't EuTiO $\mathrm{O}_{3}$ a ferroelectric in bulk?

Before we begin discussing our results we must make 
it clear in what sense we are "surprised" that $\mathrm{EuTiO}_{3}$ is not ferroelectric in bulk. First, note the similarity to the perovskite $\mathrm{SrTiO}_{3}$ : both compounds in the cubic phase have almost identical lattice constants (to two significant figures), both have nominally $\mathrm{Ti}^{4+}$ in an oxygen octahedral environment, and both have an $\mathrm{A}^{2+}$ cation on the A-site. In fact these two perovskite compounds have very similar band structures. Both have a charge transfer gap - between filled oxygen 2 p states and empty Ti d states - of similar magnitude. The only major difference is the presence of narrow $\mathrm{Eu} 4 \mathrm{f}$ bands in $\mathrm{EuTiO}_{3}$ (Fig. 2 a and $2 \mathrm{~b}$ ). (The $4 \mathrm{f}$ character of the valence band is also experimentally observed!31) These 4 f electrons, however, are well localized and shielded by the $5 \mathrm{~s}$ and $5 \mathrm{p}$ electrons. As a result, they are not expected to contribute significantly to chemical bonding. Because of these facts there is good reason to believe that the structural and dielectric properties of $\mathrm{EuTiO}_{3}$ and $\mathrm{SrTiO}_{3}$ should be quite similar.

Consistent with this conjecture is the observation that both compounds undergo a structural phase transition due to the softening of a zone-boundary, antiferrodistortive mode (corresponding to a rotation of the octahedra)!28/32 34 In $\mathrm{EuTiO}_{3}$, however, this occurs at a much higher temperature $\left(\approx 100 \mathrm{~K}\right.$ for $\mathrm{SrTiO}_{3}, \approx 300 \mathrm{~K}$ for $\left.\mathrm{EuTiO}_{3}\right)$ [36 Even more surprising is the fact that $\mathrm{SrTiO}_{3}$ displays a static dielectric constant of $\epsilon \sim 10^{4}$ at low temperature. $37 / 38$ This huge dielectric constant has been explained in a picture of a nominally unstable zone-center polar phonon mode being weakly stabilized by quantum fluctuations,,$\sqrt[3739]{39}$ and as such, is referred to as a quantum paraelectric. Indeed, first-principles calculations of the infrared-active (polar) phonons within Density Functional Theory, DFT, have shown that at the experimental lattice constant, $\mathrm{SrTiO}_{3}$ displays a weak ferroelectric instability. (All DFT studies of $\mathrm{SrTiO}_{3}$ that we know of used the most common formulation of DFT, which is a static theory where fluctuations of the nuclei, quantum or thermal, are not considered). Quantum Monte Carlo studies of a first-principles parameterized effective Hamiltonian indeed show that this ferroelectric state is suppressed by quantum fluctuations, $\frac{39}{39}$ consistent with a picture of $\mathrm{SrTiO}_{3}$ being a quantum paraelectric.

In contrast, for $\mathrm{EuTiO}_{3}$, first-principles calculations of the polar phonons within DFT at the experimental cubic lattice constant have shown that all the polar modes are quite hard, with the softest mode being $\omega \sim+70$ $\mathrm{cm}^{-1}$. It is therefore hard to imagine that $\mathrm{EuTiO}_{3}$ is close to a ferroelectric phase transition. Consistent with this is the fact that the low temperature $(\sim 5 \mathrm{~K})$ dielectric constant of $\mathrm{EuTiO}_{3}$ is two orders of magnitude smaller, $\epsilon \sim 10^{2}$, than in $\mathrm{SrTiO}_{3}{ }^{19}$ While there appears to be a "rounding off" of the dielectric constant below $\sim 10 \mathrm{~K}$ in $\mathrm{EuTiO}_{3}$, which people consider to be an observable effect of quantum fluctuations, we stress that $\mathrm{EuTiO}_{3}$ would remain a paraelectric even in their absence. This is clear from every DFT study ever conducted 22/25/32 and from the experimental determination of the Cochran fit

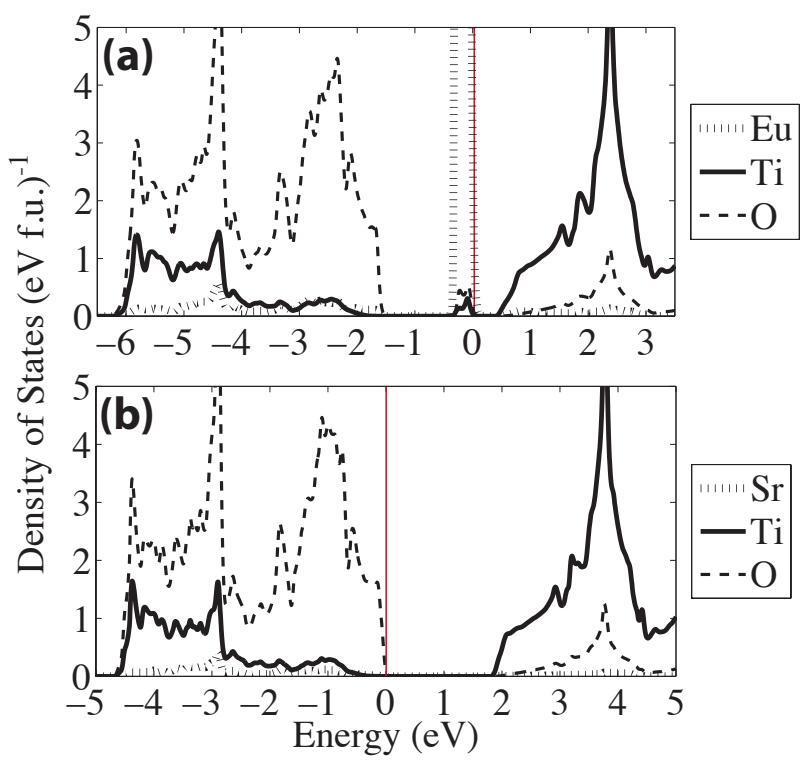

FIG. 2. (a) Density of states (DOS) of $\mathrm{EuTiO}_{3}$, calculated from first-principles. (b) DOS of $\mathrm{SrTiO}_{3}$, calculated from first-principles. The zero points of energy in the DOS plots are aligned with the highest occupied level, and are also shown by the vertical red lines.

to soft-mode frequency, which gives $\omega_{S O} \sim 75 \mathrm{~cm}^{-1}$ at zero temperature. ${ }^{[4]}$ Because of these facts we would not refer to $\mathrm{EuTiO}_{3}$ as a quantum paraelectric.

Our question should therefore be understood within the following sense: given that lattice instabilities in $\mathrm{A}^{2+} \mathrm{TiO}_{3}$ perovskites tend to be controlled by volume (baring chemistry differences, e.g., the $\mathrm{Pb}^{2+}$ lone-pair cation), and that $\mathrm{SrTiO}_{3}$ and $\mathrm{EuTiO}_{3}$ have almost identical volumes in the cubic phase, what leads to the giant hardening of the soft polar mode is $\mathrm{EuTiO}_{3}$ compared with $\mathrm{SrTiO}_{3}$ ? What seems to be the only possible explanation is that somehow the Eu $4 \mathrm{f}$ electrons have a giant effect on the lattice instabilities: dramatically decreasing (increasing) the tendency for $\mathrm{EuTiO}_{3}$ to display a ferroelectric instability (rotational instability).

In this Article we discuss the answer to these questions, thereby providing a microscopic picture of spinphonon, or more accurately spin-lattice, coupling in $\mathrm{EuTiO}_{3}$ from first principles. (Note, the physics of spinphonon coupling that we are discussing is in actuality a spin-lattice coupling, i.e., the effect of the magnetic order/correlations on the force constants, an inherently static quantity! 11] We explain our methods in Section II and give a brief background on $\mathrm{EuTiO}_{3}$ in Section III A. In Section IIIB, we explain the evolution of the polar soft-mode frequency under changing Hubbard- $U_{E u}$. We then elucidate the key role played by the hybridization of the filled $\mathrm{Eu} 4 \mathrm{f}$ states with those of the nominally empty Ti d states in Section IIIC. In Section IIID we show how this leads to a giant hardening of the polar soft mode, 
subsequently driving $\mathrm{EuTiO}_{3}$ away from ferroelectricity and rendering it a paraelectric with a small dielectric constant. In Section IIIE we explore the magnetic order dependence of this hybridization and show how it is the dominant cause of spin-lattice coupling. In Section IIIF we argue that the much stronger oxygen octahedral rotations in $\mathrm{EuTiO}_{3}$ compared to $\mathrm{SrTiO}_{3}$ also originates from the hybridization of the Eu f electrons. Finally, we conclude with a summary in Section IV]

\section{METHODS}

First-principles calculations were performed within density functional theory using the PBE-GGA exchangecorrelation functiona 40 and the Projector Augmented Wave method ${ }^{41 / 42}$ as implemented in VASP ${ }^{43 \mid 44}$ Because of the well-known deficiency of PBE-GGA in describing the localized nature of f-electrons of e.g., Eu, the DFT+U formalism is used. ${ }^{45 / 46}$ The on-site exchange $J_{E u}$ is kept fixed at $1.0 \mathrm{eV}$, while a Hubbard $-U_{E u}=6.2 \mathrm{eV}$ was found to give the best fit to experiment (where we compared the ratio of the Néel to Curie temperatures calculated within mean field theory). The value of $U_{E u}$, however, is often varied in our calculations in order to probe the physics of the system, as will be described. The cubic lattice constant is kept fixed to the experimental value of $a=3.90 \AA$. Phonon frequencies are calculated using both Density Functional Perturbation Theory and frozen phonons technique and no discrepancy is observed. We made extensive use of the Isotropy Software Package 47 and the Bilbao Crystallographic Server $\underline{48 \text { 51. Visualiza- }}$ tion of crystal structures are made using Vesta .52$]$ Maximally Localized Wannier Functions (MLWF) are calculated using the Wannier90 code. ${ }^{53 \mid 54}$

\section{RESULTS}

\section{A. Background}

The cubic crystal structure of perovskite $\mathrm{EuTiO}_{3}$ is shown in Fig. 11a. Rotations of oxygen octahedra, which are known to exist in bulk $\mathrm{EuTiO}_{3}$, were recently shown to have a strong effect on magnetism. $\frac{29|32| 33|55| 57}{57}$ In particular, they alter the magnetic exchange interactions in a way that strongly favors antiferromagnetism over ferromagnetism. Additionally, rotations tend to suppress the tendency towards ferroelectricity ${ }^{[29] 33}$ In the epitaxial strain phase diagram of $\mathrm{EuTiO}_{3}$ this results in an increase in the critical strain value necessary to induce a transition form the paraelectric-antiferromagnetic phase to the ferroelectric-ferromagnetic phase. For tensile strain, however, this increase in critical strain is almost canceled by the larger value of Hubbard- $U_{E u}$, which lowers the critical strain, that is now necessary to give a reasonable fit of the magnetic exchange interactions in the presence

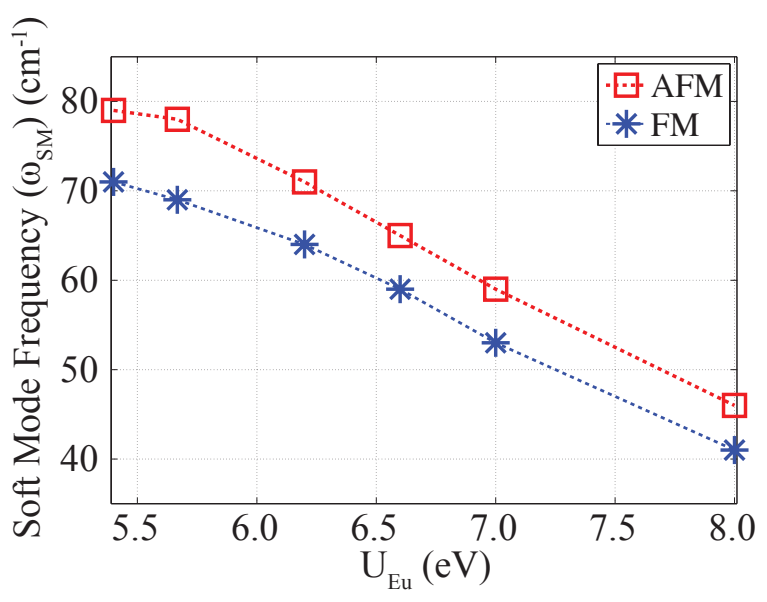

FIG. 3. Polar soft-mode frequency, $\omega_{S M}$, vs. the on-site interaction $U_{E u}$ on Eu $4 \mathrm{f}$ orbitals. Red squares and blue asterisks denote the frequencies calculated in AFM and FM states respectively.

of rotations to experiment (see Ref. 29 for a complete discussion).

Magnetism in $\mathrm{EuTiO}_{3}$ stems from the half filled $4 \mathrm{f}$ shell of the Europium cation, which have 7 electrons in a highspin state. These well localized spins order in a collinear G-type antiferromagnetic fashion (Fig. 1p) so that the spin of each Eu cation is opposite to all of its nearest neighbors. As seen in the density of states (DOS) in Fig. $2 \mathrm{a}$, there is a wide charge transfer gap between the the occupied O-p states and the conduction band that consists of unoccupied Ti-d states. The half-occupied Eu $4 \mathrm{f}$ states form narrow bands below the Fermi level in this charge transfer gap. There is very little hopping between the Eu$4 \mathrm{f}$ orbitals and the neighboring cations, since the radii of the $4 \mathrm{f}$ orbitals are much smaller than that of the $5 \mathrm{~s}$ or $5 \mathrm{p}$ orbitals. This is the reason that the Néel temperature is low, and the Eu-f bands are narrow. (The Néel temperature is further lowered because of the competition between ferromagnetic and antiferromagnetic exchange interactions.) The Hubbard-U applied on the Eu-f states $\left(U_{E u}\right)$ shifts the energy level of the narrow Eu-f bands, and hence determines the gap between them and the Ti $\mathrm{d}$ or $\mathrm{O}$ p bands.

\section{B. An Intriguing thought experiment: Polar mode frequency versus Hubbard-U}

To begin unraveling the mechanism behind the soft mode behavior of $\mathrm{EuTiO}_{3}$, we perform a thought experiment where we calculate the frequencies, $\omega_{S M}$, of the polar phonons of cubic (space group $\mathrm{Pm} \overline{3} \mathrm{~m}$ ) $\mathrm{EuTiO}_{3}$ from first principles, for several different values of the Hubbard-U (applied to the Eu-f states, $U_{E u}$ ). This is plotted in Fig. 3. Note that the frequency at a value of $U_{E u} \sim 6 \mathrm{eV}$ reproduces well the experimental value, 
$\omega_{S M} \sim 75 \mathrm{~cm}^{-1}$, determined from a Cochran fit .58

There are two clear trends in Fig. 3 (i) as is now wellknown, $\omega_{S M}$ is lower in the ferromagnetic (FM) state, which explains ${ }^{22}$ an increase in the ionic contribution to the dielectric constant,,$[59] \epsilon_{\text {ion }} \sim 1 / \omega_{S M}^{2}$, under external magnetic field, and (ii) $\omega_{S M}$ depends sensitively on $U_{E u}$. With regards to the latter, notice how a relatively modest increase in $U_{E u}$ greatly decreases $\omega_{S M}$. This is surprising as the polar soft-mode of $\mathrm{EuTiO}_{3}$ is driven by the off-centering of the $\mathrm{Ti}^{4+}$ cation, i.e., B-site driven, in a second order Jahn-Teller like process 60 (we elaborate on this below), and therefore it is not expected to depend so sensitively on the energy of the Eu bands, or $U_{E u}$. Furthermore, the Hubbard- $U_{E u}$ acts only on the $4 \mathrm{f}$ shell of the Eu ion, which has a smaller radius than the fully occupied $5 \mathrm{~s}$ and $5 \mathrm{p}$ shells, making a direct effect on the phonon frequencies less likely.

These observations, along with the fact that magnetism originates from the unpaired electrons on Eu, suggests that the Eu-f states may play a role in the origin of spin-lattice coupling. To probe this further, next we take a closer look at the spin-dependent hybridization of Eu-f electrons with other orbitals and with its effect on the soft-mode behavior.

\section{Magnetic order control of Eu-f/Ti-d/O-p hybridization}

Despite the small radii of the $\mathrm{Eu} 4 \mathrm{f}$ orbitals, there are no bands purely of Eu character. This is seen in the site-projected density of states (DOS) as shown in Fig. 2 a. The DOS peak right below the Fermi level is of dominantly Eu-f character, i.e., the wavefunctions in the energy window corresponding to this peak are mostly localized on the $\mathrm{Eu}$ ions, and have the symmetry of $\mathrm{f}$ states. There is, however, a small but non-zero contribution from $\mathrm{Ti}$ and $\mathrm{O}$ ions to this peak as well, indicating that the Eu-f states hybridize with both $\mathrm{Ti}$ and $\mathrm{O}$ atomic orbitals. ${ }^{30}$ This becomes strikingly clear by considering maximally localized Wannier functions (MLWFs) $\stackrel{53}{5}$ In Figure 4, we show two examples of occupied Eu $\mathrm{f}$ MLWFs, $f_{z y^{2}}$ and $f_{x y z}$ Wannier orbitals. 64 The cubic harmonics corresponding to these orbitals are proportional to $z\left(4 y^{2}-x^{2}-z^{2}\right)$ and $x y z$ respectively.

Of particular interest is the Eu-ion's $f_{x y z}$ Wannier orbital, Fig. $4 \mathrm{p}$, which has lobes directed towards the $\mathrm{Ti}$ cation. Notice that the MLWF is mostly localized around the ion's core. There is, however, a small, but nonzero, weight around the neighboring $\mathrm{Ti}$ ions. This is an explicit sign that this Eu state hybridizes with a nominally empty Ti-d state(s). This hybridization is important for several reasons, e.g., it has been shown previously that it leads to a superexhange interaction mediated through the Ti cations. 113065

Our interest here is in the fact that this component of the MLWF can be thought as representing the partial occupation of the Ti-d orbitals. It turns out that the de- pendance of this hybridization on a Hubbard- $U$ applied to the Eu-f states, $U_{E u}$, brings out the underlying physics of spin-phonon coupling in $\mathrm{EuTiO}_{3}$. In Fig. 5, we plot the total charge within the Ti-d manifold of states due to the hybridization with Eu-f states $\left(\sigma_{T i}\right)$ as a function of $U_{E u}$, considering both the ground state G-type antiferromagnetic and ferromagnetic spin configurations. We obtain $\sigma_{T i}$ by integrating the DOS projected onto the Ti-d shell over the energy window corresponding to the Eu-f bands (between $\approx-0.5$ and $\approx 0.0 \mathrm{eV}$ ). This quantity, $\sigma_{T i}$, is also related to the weight of the Eu-f MLWFs localized on a $\mathrm{Ti}$ site seen in Fig. $4 \mathrm{~b}$. Although $\sigma_{T i}$ is small, there are two clear trends that are evident in Fig. 5.: (i) $\sigma_{T i}$ decreases with increasing $U_{E u}$ and (ii) $\sigma_{T i}$ is larger in the AFM state compared to the FM one.66

The latter will be explained in the proceeding Section while the former, a change in the amount of hybridization with increasing $U_{E u}$, is not surprising. Adding a Coulomb interaction, U, to DFT causes the corresponding orbitals to become more local. Increasing $U_{E u}$ makes it energetically favorable for electrons to remain localized in Eu-f orbitals. Also, as observed in Ref. 23, the Eu-f bands move away in energy from the conduction band when $U_{E u}$ is increased. As a result, the hybridization between the Eu-f and the Ti-d states decreases. This is also clearly visible in Fig. 6, where the $f_{x y z}$ MLWF is plotted for different values of $U_{E u}$. As the Coulomb interaction increases, the lobes localized near the Ti cation get smaller and eventually disappear, consistent with a decreasing $\sigma_{T i}$.

\section{The suppression of ferroelectricity}

Ferroelectricity in prototypical perovskite ferroelectrics such as $\mathrm{BaTiO}_{3}$ originates from a "cross-gap" hybridization 67 of a cation's empty orbitals at the bottom of the conduction band (typically either a transition metal cation's d-orbitals, e.g., $\mathrm{Ti}^{4+}$, or a lone pair active cation's p-orbitals, e.g., $\mathrm{Bi}^{3+}$ ) and the occupied p states of the oxygens at the top of the valence band. This mechanism can be thought of as a second order Jahn-Teller like process 60 This is the mechanism for ferroelectricity in strained $\mathrm{EuTiO}_{3}$. Here, the displacement of a Ti cation towards one of the oxygens increases the Ti-3d/O$2 \mathrm{p}$ hybridization, thereby moving the hybridized empty states to higher energies, while lowering the energy of the hybridized occupied states. This "rehybridization" leads to a second order energy gain favoring ferroelectricity. If, however, the transition metal d states are partially occupied, there is an extra energy cost associated with moving these states to higher energies, and the tendency towards ferroelectricity is reduced $[68$ This argument has been mentioned often in the context of the incompatibility of ferroelectricity with B-site magnetism,, 69 and is central to both the suppression of ferroelectricity and the origin of spin-lattice coupling in $\mathrm{EuTiO}_{3}$. But first we must understand why $\sigma_{T i}$ is larger in the AFM state 

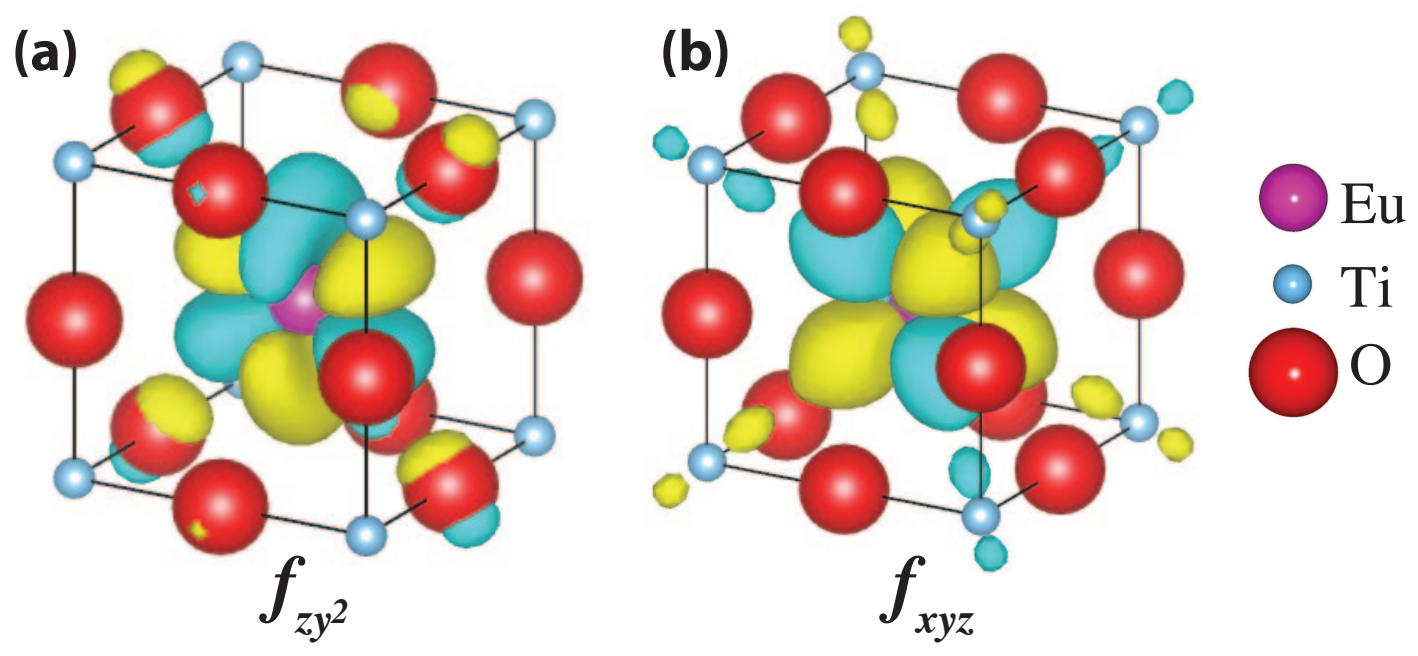

FIG. 4. Two examples of maximally localized Wannier functions of Eu f electrons in $\operatorname{EuTiO}_{3}$. (a) $f_{z y^{2}} \sim z\left(4 y^{2}-z^{2}-x^{2}\right)$, (b) $f_{x y z} \sim x y z$. Yellow and green parts of the Wannier Function correspond to isosurfaces of opposite sign, and the Europium ion is in the center of the cubic cell.

than in a state with parallel spins (FM).

Consider the $\mathrm{Eu}-\mathrm{Ti}-\mathrm{Eu}$ exchange pathway along the [111] direction. In bulk $\mathrm{EuTiO}_{3}$ these Eu cations have a strong AFM interaction, which leads to the observation of (predominantly) G-type magnetic order $29[55[56$ In Fig. $7 \mathrm{a}$, the $f_{x y z}$ orbitals on two neighbor spin-polarized $\mathrm{Eu}^{2+}$ cations, and the $d_{(x+y+z)^{2}}$ orbital on the intermediate non-magnetic $\mathrm{Ti}^{4+}$ cation are shown. (This particular $d$ orbital has lobes directed towards both Eu cations and therefore will have the largest hopping to/from the $f_{x y z}$ orbitals.)

First, imagine that the Eu spins were aligned parallel

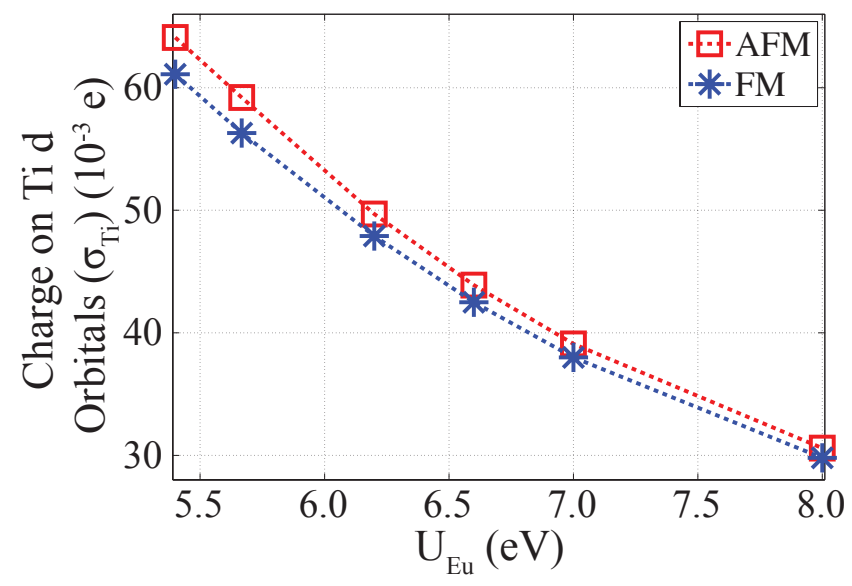

FIG. 5. Charge on Ti d orbitals due to hybridization of the Eu f states $\left(\sigma_{T i}\right)$ versus Hubbard- $U_{E u}$ used in DFT $+\mathrm{U}$ calculations. Red squares and blue asterisks denote values in AFM and FM states respectively.
(FM), rather than in the observed AFM configuration. In Fig. 7b, we sketch the energy levels of the three orbitals in this state. Notice that an electron from either Eu cation is allowed by symmetry to hop to the Ti cation (Fig. 7p and d), but that the higher order process where both electrons hop to the Ti atom simultaneously (Fig. 7 e) is not allowed due to Pauli exclusion principle. Next consider the same process but with the spins aligned antiparallel (AFM) (Fig. 7f). Now, in addition to the two individual hopping processes, Fig. $7 \mathrm{~g}$-h, the correlated hopping process in Fig. 77 is allowed, leading to a larger hybridization, thus, a larger $\sigma_{T i}$ in the AFM state. Combining the physics represented in Figure 7 with that of the rehybridization mechanism of ferroelectricity leads to a straightforward explanation for the suppression of ferroelectricity and the origin of spin-lattice coupling in $\mathrm{EuTiO}_{3} \cdot{ }^{70}$

As a thought experiment, initially consider bulk $\mathrm{EuTiO}_{3}$ in which the Eu-f/Ti-d hybridization was removed. One way this can be done from first-principles is by putting the f-electrons in the core of the PAW potential. In this case, the Ti-d states are essentially empty and are free to hybridize with the O-2p states as the $\mathrm{Ti}^{4+}$ cations move off-center, creating a polar lattice distortion is a second-order Jahn-Teller process. In this case, $\mathrm{EuTiO}_{3}$ should have a ferroelectric instability as in $\mathrm{SrTiO}_{3}$. Our calculations directly confirm this. Turning on the Eu-f/Ti-d hybridization, by moving the f-eletroncs from the core of the PAW potential to the valence, increases the occupancy of the Ti d states. This lowers the energy gained from the 2nd order Jahn-Teller thereby decreasing the tendency towards ferroelectricity, and thus hardening the polar soft-mode. This not only explains the suppression of ferroelectricity in $\mathrm{EuTiO}_{3}$ but also our previous result shown in Fig. 3 (another "dial" 


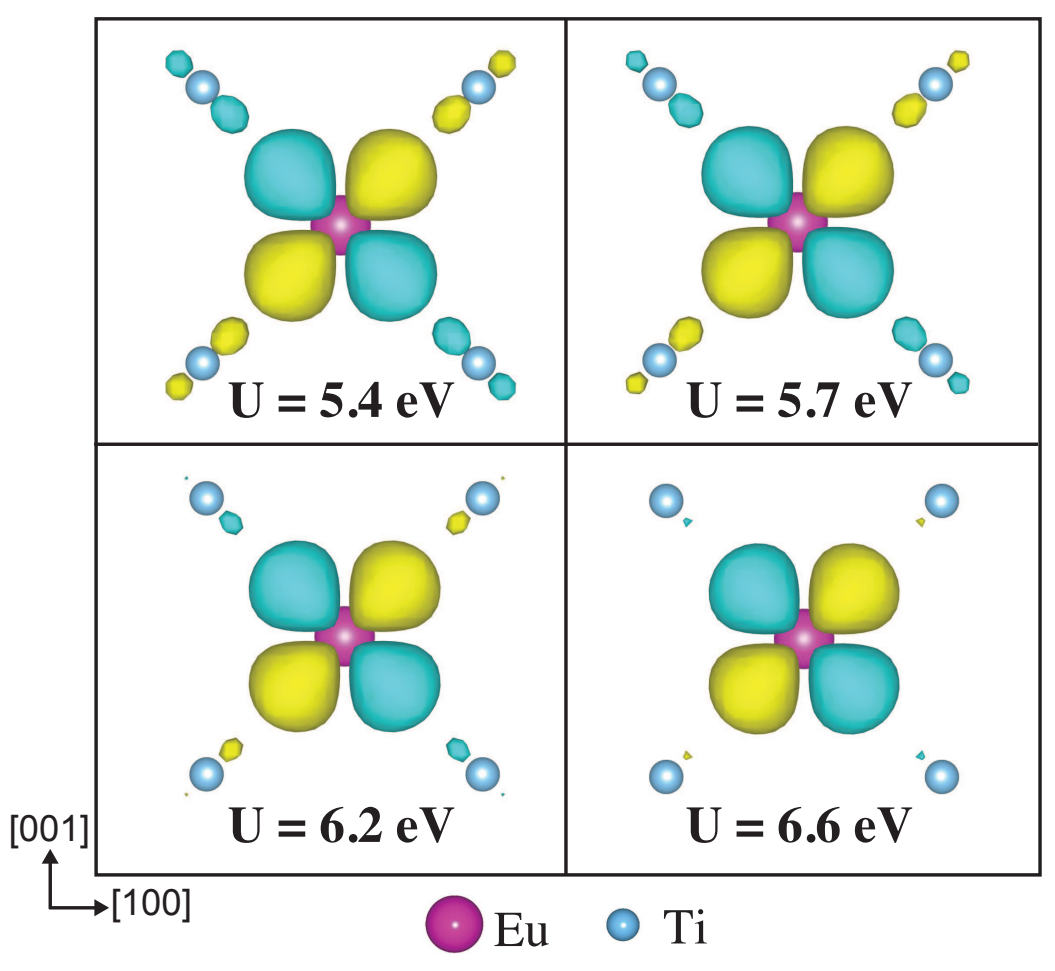

FIG. 6. The Eu $f_{x y z}$ MLWF for different values of $U_{E u}$. For simplicity, the oxygen ions are not shown on the figure.

one can turn to remove, albeit partially, the Eu-f/Ti-d hybridization, and thus increase the tendency towards ferroelectricity, is to increase the Hubbard-U applied to the Eu f states, $U_{E u}$.)

In order to lend support for this scenario, the self force constants, $\tilde{C}$ (i.e., the second derivatives of the total energy with respect to the corresponding ionic displacements) of the 4 symmetry adapted modes of the infraredactive (IR-active) irrep are plotted as a function of $U_{E u}$ in Fig. 8. First note that only $\tilde{C}_{T i}$ and $\tilde{C}_{O \|}$, which are the only symmetry adapted modes that lead to a first order change in Ti-O distances, decrease significantly with increasing $U_{E u}$, while $\tilde{C}_{E u}$ actually increases. Therefore, the softening of $\omega_{S M}$ with increasing $U_{E u}$ primarily comes from the softening of the relative motion of $\mathrm{Ti}$ moving against $O \|$ and not from the Eu motion. These observations support the claim that the phonon softening with increasing $U_{E u}$ is a consequence of decreasing Eu-Ti hybridization.

One question that is natural to ask at this point is whether the emergence of ferroelectricity in $\mathrm{EuTiO}_{3}$ films under biaxial strain ${ }^{22128}$ is related to a decrease in the Euf/Ti-d hybridization. In order to check this possibility, we calculated the DOS and $\sigma_{T i}$ for $\mathrm{EuTiO}_{3}$ under biaxial strain, not taking into account oxygen octahedral rotations or polarization. The results (not shown) indicate that while $\sigma_{T i}$ indeed depends on the strain, the change in $\sigma_{T i}$ for reasonable values of strain is no larger than few percent. Thus the emergence of ferroelectricity in $\mathrm{EuTiO}_{3}$ under strain is likely to be of similar nature to that in strained $\mathrm{SrTiO}_{3}$ and $\mathrm{CaMnO}_{3}{ }^{71 / 72}$

\section{E. The mechanism of spin-lattice coupling and the origin of ferromagnetism in strain-induced ferroelectric $\mathrm{EuTiO}_{3}$}

If the spins in $\mathrm{EuTiO}_{3}$ could be aligned in a parallel direction, e.g., in the presence of a strong magnetic field, the system would respond by decreasing the Euf/Ti-d hybridization, which would subsequently decrease the occupancy of the Ti-d states, $\sigma_{T i}$, and result in a softening of the polar soft-mode. As a result, $\tilde{C}_{T i}$ and $\tilde{C}_{O \|}$ should (and do) have a significant FM-AFM splitting, while $\tilde{C}_{E u}$ and $\tilde{C}_{O \perp}$ have none, as clearly seen in Fig. 8. Also note that the splittings of $\tilde{C}_{T i}$ and $\tilde{C}_{O \|}$ decrease with increasing $U_{E u}$, since $\sigma_{T i}$ decreases. This is the microscopic origin of the spin-phonon observations of Katsufuji and Takagi. (Note that the AFM-FM splitting of $\omega_{S M}$ does not decrease significantly with $U_{E u}$, Fig. 3 , because the eigenvector changes.)

Now imagine that the tendency towards ferroelectricity is greatly increased so as to dominate over the electronic energy gained from the Eu-f/Ti-d hybridization. The system would respond by decreasing the occupancy of the Ti-d states, $\sigma_{T i}$, so as to further increase the energy gain 

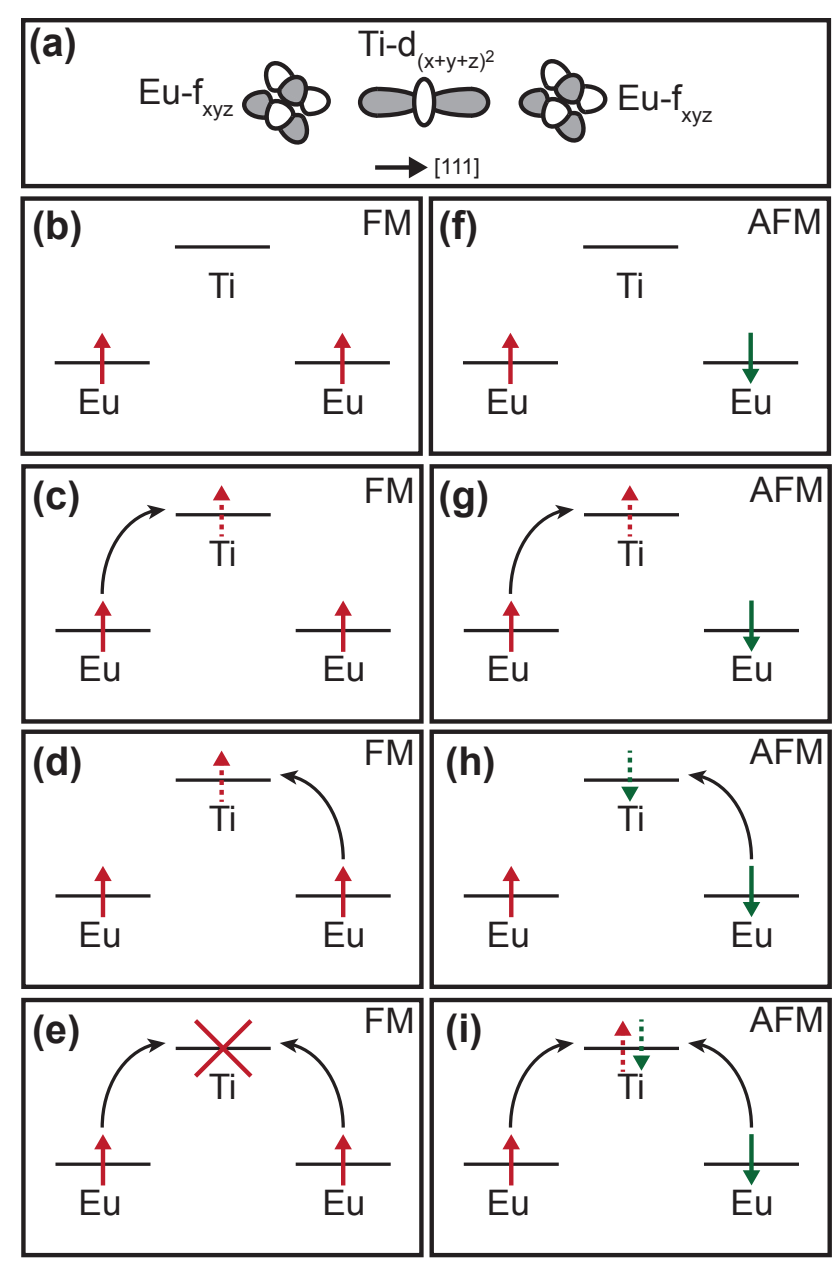

FIG. 7. (a) Sketch of the $f_{x y z}$ orbitals on $3^{\text {rd }}$ neighbor Eu ions and the intermediate Ti ion's $d_{(x+y+z)^{2}}$ orbital. (b) Energy levels of the three orbitals in the FM state. Lowest lying excitations where an electron hops onto the Ti cation, (c) and (d), are allowed, but not both the electrons can hop at the same time because of Pauli exclusion (e). However, in the AFM state, (f), not only the lowest excitations (g) and (h) but also the correlated hopping sketched in (i) is allowed.

from the polar lattice distortion. This is accomplished by decreasing the Eu-f/Ti-d hybridization, thereby promoting FM interactions between the spins.

As an additional cross-check, in Fig. 9 we plot $\tilde{C}_{T i}$ as a function of $\sigma_{T i}$. The self force constant of $\mathrm{Ti}$ is seen to have an almost linear dependence on $\sigma_{T i}$ and more importantly, it does not depend on the particular magnetic order, FM or AFM. This universal behavior indicates that the dominant factor determining the change in $\tilde{C}_{T i}$, and therefore $\omega_{S M}$, is indeed $\sigma_{T i}{ }^{[73}$

As a final check of the validity of our arguments we add a Hubbard-U on the Ti d orbitals as well. Increasing the energy cost of occupying Ti d states suppresses both the spin-phonon coupling and the dependence of $\omega_{S M}$ on $U_{E u}$ as expected. The strong dependence of spin- phonon coupling to the energy of Ti d states explains why similar spin-lattice physics has not been observed in other compounds similar to $\mathrm{EuTiO}_{3}$, such as $\mathrm{EuZrO}_{3}{ }^{74}$ (Our calculations for cubic $\mathrm{EuZrO}_{3}$ and $\mathrm{EuHfO}_{3}$ indicate that the spin-phonon coupling in these materials is smaller than the numerical error, in line with our arguments.)

\section{F. Oxygen octahedral rotations}

The second question we posed in the introduction concerned the much stronger energy scale associated with ro-

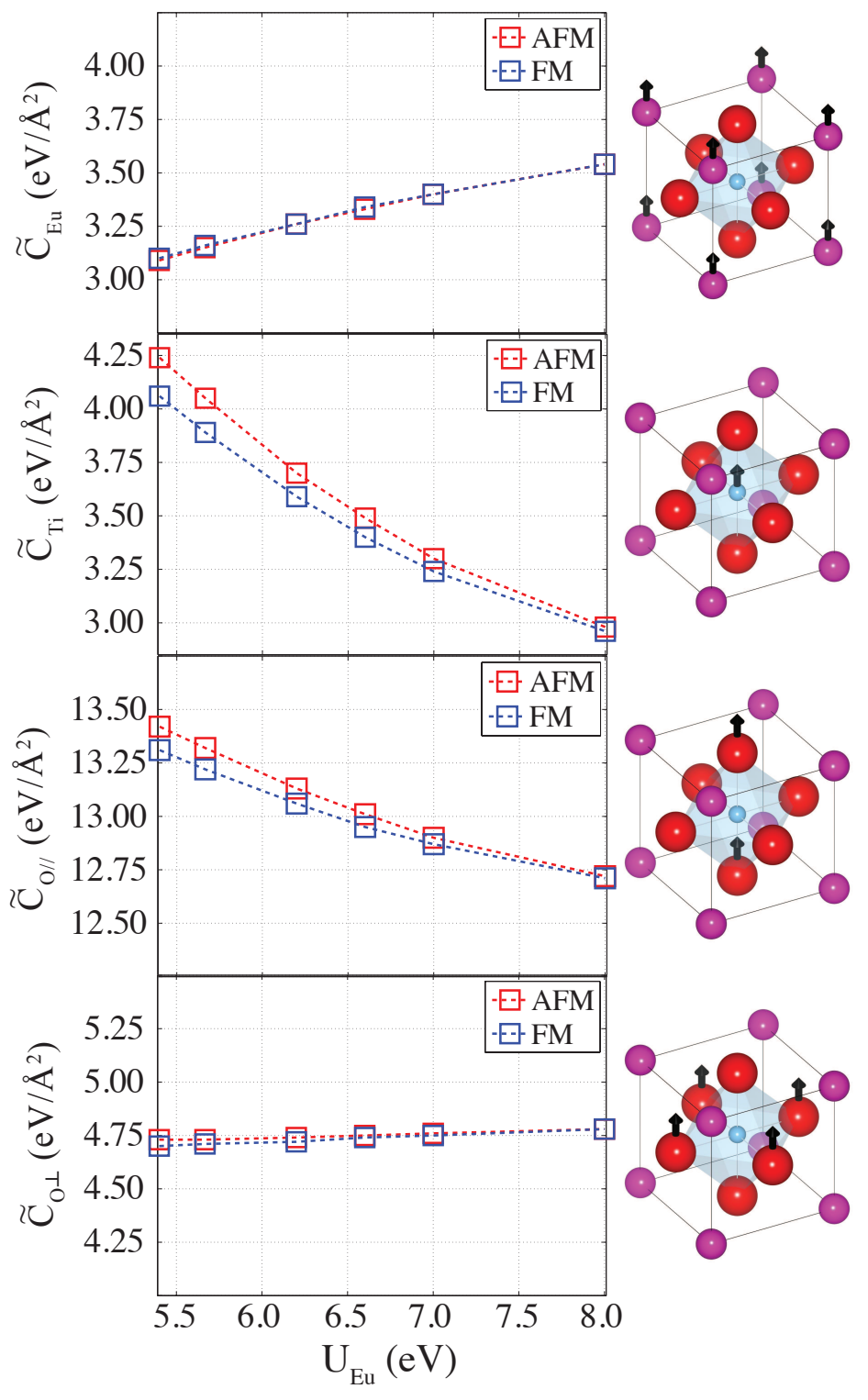

FIG. 8. Self force constants $(\tilde{C})$ for the four $\Gamma$ point symmetry adapted modes (left) and the corresponding displacement patterns (right). Red squares and blue asterisks denote the force constants in AFM and FM states respectively. 


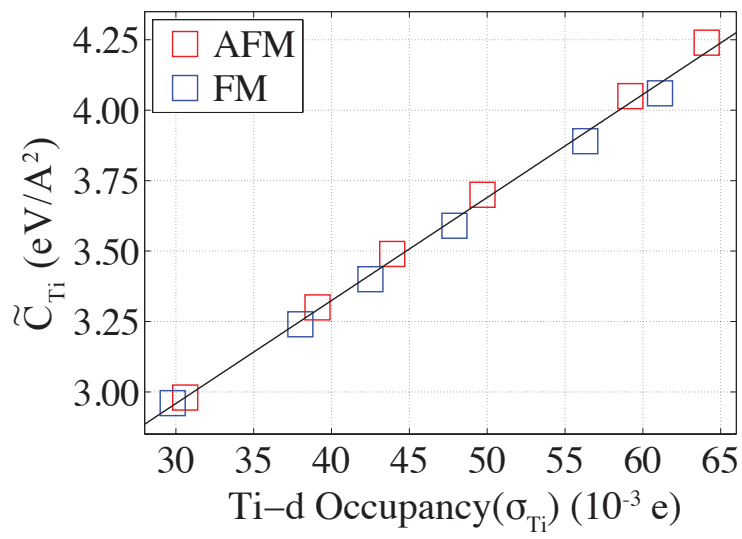

FIG. 9. Self force constant of Ti ion $\left(\tilde{C}_{T i}\right)$ as a function of $\sigma_{T i}$ (the charge on $\mathrm{Ti} \mathrm{d}$ shell due to hybridization with $\mathrm{Eu}$ $\mathrm{f}$ states). Red and blue curves denote values calculated in AFM and FM states respectively. Black line is a best fit to the data. Note that the data on this plot can be extracted from Fig. 5 and 8 .

tations of the octahedra in $\mathrm{EuTiO}_{3}$ compared with those in $\mathrm{SrTiO}_{3}$. The experimentally measured octahedral rotation angle is also much larger in $\mathrm{EuTiO}_{3}\left(3.6^{\circ}\right)$ com-

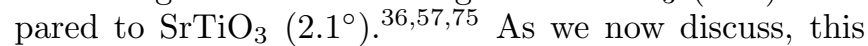
also can be answered by considering the effect of Eu-f states.

Woodward, in his seminal work, ${ }^{76}$ showed that covalent bonding between the A-site cation and the oxygen anions has a stabilizing effect on the oxygen octahedral rotations in perovskites. The octahedral rotations change the coordination environment of the A-site and as a result significantly alter the covalent bonding strength and hybridization between the A-site cation and the oxygens. Akamatsu et al. pointed out another important effect of octahedral rotations in $\mathrm{EuMO}_{3}(\mathrm{M}=\mathrm{Ti}, \mathrm{Zr}, \mathrm{Hf})$ perovskites; $\sqrt[56]{6}$ they increase the overlap between the Eu$\mathrm{f}$ and B-site $\mathrm{d}$ orbitals. This results in an enhanced hybridization between these orbitals, which can also be seen explicitly in the charge density $\stackrel{56}{5}$ Just as the increased A-O covalency stabilizes octahedral rotations, this increased $\mathrm{A}-\mathrm{B}$ hybridization also lowers the energy and hence stabilizes the rotational lattice distortion. This explains the stronger rotations observed in $\mathrm{EuTiO}_{3}$ compared to $\mathrm{SrTiO}_{3}$.

To help shed light on this observation, in Fig. 10a we plot the phonon frequency associated with the $\mathrm{R}$ point rotation mode in cubic $\mathrm{EuTiO}_{3}$ as a function of $U_{E u}$. With increasing $U_{E u}$, the f electrons become more localized on the Eu ion, and as a result the stabilizing effect of the Eu-f/Ti-d hybridization decreases. This in turn results in the rotation soft mode becoming more stable (the magnitude of the imaginary frequency decreases). A similar trend is also observed in the ground state octahedral rotation angles reported in Fig. 10p; the rotation angle
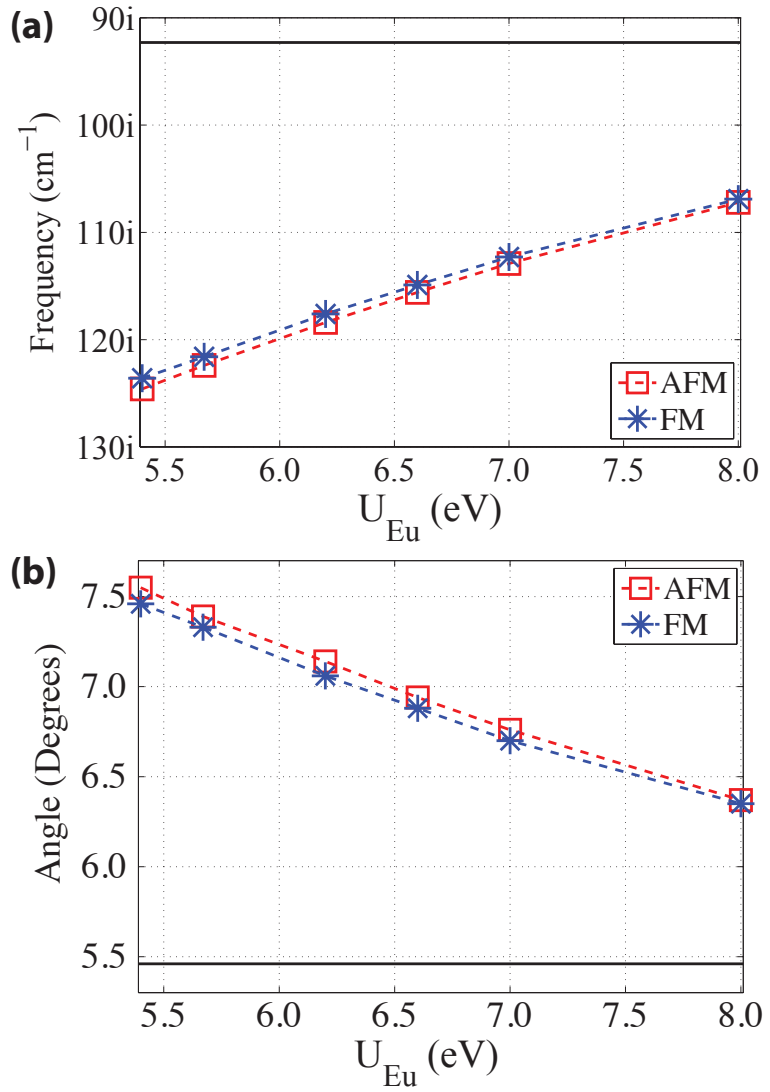

FIG. 10. (a) R point rotation soft mode frequency as a function of $U_{E u}$ in the FM and the AFM states and the cubic structure with lattice constant fixed to $3.90 \AA$. The horizontal black line corresponds to the soft mode frequency in $\mathrm{SrTiO}_{3}$, calculated with the same settings. (b) Octahedral rotation angle, obtained by relaxing the ions in fixed cubic cell, as a function of $U_{E u}$ in the FM and the AFM states. The horizontal black line corresponds to the rotation angle in $\mathrm{SrTiO}_{3}$, calculated with the same settings. Lines connecting the data points are provided to guide the eye.

decreases with increasing $U_{E u}$, approaching the value of $\mathrm{SrTiO}_{3}$. In other words, in terms of the octahedral rotations, the behavior of $\mathrm{EuTiO}_{3}$ gets closer to that of $\mathrm{SrTiO}_{3}$ with increasing $U_{E u}$. ${ }^{77}$ Note that one should be able to ignore the effect of changing $U_{E u}$ on the Eu-O covalent bonding as the unoccupied Eu-f states lie at energies much higher than the Fermi level, above the empty Eu-s states. The change in the rotation angle with $U_{E u}$, therefore, should be attributed solely to the changes in the Eu-f/Ti-d hybridization.

We also performed similar calculations to compare $\mathrm{EuZrO}_{3}$ with $\mathrm{SrZrO}_{3}$, using the same rotation pattern as $\mathrm{EuTiO}_{3}$. The rotation angle difference between these two zirconates is as small as $\approx 0.3^{\circ}$. This is because $\mathrm{EuZrO}_{3}$ has a much larger band gap than $\mathrm{EuTiO}_{3}$, and as a result there is not a significant hybridization between the Eu-f and the Zr-d states that strengthens the octa- 
hedral rotations. The same applies to $\mathrm{EuHfO}_{3}$, which has an octahedral rotation angle of $11.9^{\circ}$; merely $\sim 0.3^{\circ}$ degrees larger than $\mathrm{SrHfO}_{3}$.

\section{SUMMARY}

In summary, by employing $\mathrm{DFT}+\mathrm{U}$ calculations and MLWFs, we elucidated the key role played by the Euf/Ti-d hybridization in $\mathrm{EuTiO}_{3}$. The resultant charge transferred to the Ti-d states $\left(\sigma_{T i}\right)$ drives the system away from a ferroelectric (or quantum paraelectric) phase, and causes a dielectric behavior manifestly different from that of $\mathrm{SrTiO}_{3}$. The dependence of $\sigma_{T i}$ on the magnetic order causes the polar soft-mode frequency to depend on the magnetic state. This is the leading contribution to spin-phonon coupling in $\mathrm{EuTiO}_{3}$. Octahedral rotations are also affected from Eu f states' hybridization with Ti d orbitals, and are stronger in $\mathrm{EuTiO}_{3}$ compared to $\mathrm{SrTiO}_{3}$ as a result.

These results underline the importance of rare earth $f$ electrons in the lattice dynamics and dielectric behavior of TM oxides. ${ }^{78}$ While the present study is focused on EuTiO ${ }_{3}$, similar effects could exist in other compounds as well. Taking advantage of the hybridization of rare earth cation with the TM ion might be used as a new knob to tune the system away or close to the ferroelectric transition or perhaps to a quantum critical point.

\section{ACKNOWLEDGMENTS}

We acknowledge fruitful discussions with T.A. Arias, N.A. Benedek, S. Kamba and A. Stroppa. This work is supported by the DOE-BES under Grant No. DESCOO02334.
1 C.-W. Nan, M. I. Bichurin, S. Dong, D. Viehland, and G. Srinivasan, Journal of Applied Physics 103, 031101 (2008).

2 L. W. Martin and D. G. Schlom, Current Opinion in Solid State and Materials Science 16, 199 (2012).

3 L. W. Martin and R. Ramesh, Acta Materialia 60, 2449 (2012).

4 Q. He, E. Arenholz, A. Scholl, Y.-H. Chu, and R. Ramesh, Current Opinion in Solid State and Materials Science 16, 216 (2012).

5 M. Cohen, Science 261, 307 (1993).

6 A. Franceschetti and A. Zunger, Nature 402, 60 (1999).

7 N. A. Spaldin and W. E. Pickett, Journal of Solid State Chemistry 176, 615 (2003).

8 J. Hafner, C. Wolverton, and G. Ceder, MRS Bulletin 31, 659 (2006).

${ }^{9}$ N. A. Benedek and C. J. Fennie, Phys. Rev. Lett. 106, 107204 (2011).

10 E. Bousquet and N. Spaldin, Phys. Rev. Lett. 107, 197603 (2011).

11 T. Birol, N. A. Benedek, H. Das, A. L. Wysocki, A. T. Mulder, B. M. Abbett, E. H. Smith, S. Ghosh, and C. J. Fennie, Current Opinion in Solid State and Materials Science 16, 227 (2012).

12 S. Picozzi and A. Stroppa, European Physical Journal B 85, 1 (2012).

13 Z. P. Yin and G. Kotliar, Europhysics Letters 101 (2013).

14 J. H. Lee and K. M. Rabe, Phys. Rev. Lett. 104, 207204 (2010).

15 J. H. Lee and K. M. Rabe, Phys. Rev. Lett. 107, 067601 (2011).

16 G. Giovannetti, S. Kumar, C. Ortix, M. Capone, and J. van den Brink, Phys. Rev. Lett. 109, 107601 (2012).

17 T. McGuire, M. Shafer, R. Joenk, H. Alperin, and S. Pickart, Journal of Applied Physics 37, 981 (1966).

18 C.-L. Chien, S. DeBenedetti, and F. D. S. Barros, Physical Review B 10, 3913 (1974).

19 T. Katsufuji and H. Takagi, Physical Review B 64, 054415 (2001).
20 J. H. Lee and K. M. Rabe, Phys. Rev. B 84, 104440 (2011).

21 J. Hong, A. Stroppa, J. Íñiguez, S. Picozzi, and D. Vanderbilt, Physical Review B 85, 054417 (2012).

22 C. J. Fennie and K. M. Rabe, Phys. Rev. Lett. 97, 267602 (2006).

23 R. Ranjan, H. S. Nabi, and R. Pentcheva, Journal of Physics: Condensed Matter 19, 406217 (2007).

24 S. Kamba, D. Nuzhnyy, P. Vaněk, M. Savinov, K. Knížek, Z. Shen, E. Šantavá, K. Maca, M. Sadowski, and J. Petzelt, Europhysics Letters 80, 27002 (2007).

25 S. Kamba, V. Goian, M. Orlita, D. Nuzhnyy, J. Lee, D. Schlom, K. Rushchanskii, M. Ležaić, T. Birol, C. Fennie, et al., Physical Review B 85, 094435 (2012).

26 R. E. Newnham, Acta Crystallographica Section A 54, 729 (1998).

27 Y. Tokura, Reports on Progress in Physics 69, 797 (2006).

28 J. H. Lee, L. Fang, E. Vlahos, X. Ke, Y. W. Jung, L. F. Kourkoutis, J.-W. Kim, P. J. Ryan, T. Heeg, M. Roeckerath, et al., Nature 466, 954 (2010).

29 P. Ryan, J.-W. Kim, T. Birol, P. Thompson, J.-H. Lee, X. Ke, P. Normile, E. Karapetrova, P. Schiffer, S. Brown, C. Fennie, and D. Schlom, Nature Communications 4, 1334 (2013).

${ }^{30}$ H. Akamatsu, Y. Kumagai, F. Oba, K. Fujita, H. Murakami, K. Tanaka, and I. Tanaka, Physical Review B 83, 214421 (2011).

31 T. Kolodiazhnyi, M. Valant, J. R. Williams, M. Bugnet, G. A. Botton, N. Ohashi, and Y. Sakka, Journal of Applied Physics 112, 083719 (2012).

32 K. Z. Rushchanskii, N. A. Spaldin, and M. Ležaić, Physical Review B 85, 104109 (2012).

33 Y. Yang, W. Ren, D. Wang, and L. Bellaiche, Physical Review Letters 109, 267602 (2012).

34 D. S. Ellis, H. Uchiyama, S. Tsutsui, K. Sugimoto, K. Kato, D. Ishikawa, and A. Q. R. Baron, Phys. Rev. B 86, 220301 (2012).

35 P. A. Fleury, J. F. Scott, and J. M. Worlock, Phys. Rev. Lett. 21, 16 (1968). 
36 M. Allieta, M. Scavini, L. J. Spalek, V. Scagnoli, H. C. Walker, C. Panagopoulos, S. S. Saxena, T. Katsufuji, and C. Mazzoli, Phys. Rev. B 85, 184107 (2012).

37 K. A. Müller and H. Burkard, Phys. Rev. B 19, 3593 (1979).

38 H. Weaver, Journal of Physics and Chemistry of Solids 11, 274 (1959).

39 W. Zhong and D. Vanderbilt, Phys. Rev. B 53, 5047 (1996).

40 J. P. Perdew, K. Burke, and M. Ernzerhof, Phys. Rev. Lett. 77, 3865 (1996).

41 P. E. Blöchl, Phys. Rev. B 50, 17953 (1994).

42 G. Kresse and D. Joubert, Phys. Rev. B 59, 1758 (1999).

43 G. Kresse and J. Furthmüller, Phys. Rev. B 54, 11169 (1996).

44 G. Kresse and J. Furthmuller, Computational Materials Science 6, 15 (1996).

45 V. I. Anisimov, J. Zaanen, and O. K. Andersen, Phys. Rev. B 44, 943 (1991).

46 A. I. Liechtenstein, V. I. Anisimov, and J. Zaanen, Phys. Rev. B 52, R5467 (1995).

47 H. Stokes, D. Hatch, and B. Campbell, "Isotropy," (2007).

${ }^{48}$ M. I. Aroyo, A. Kirov, C. Capillas, J. M. Perez-Mato, and H. Wondratschek, Acta Crystallographica Section A 62, 115 (2006).

49 M. Aroyo, J. Perez-Mato, C. Capillas, E. Kroumova, S. Ivantchev, G. Madariaga, A. Kirov, and H. Wondratschek, Zeitschrift fur Kristallographie 221, 15 (2006).

50 M. I. Aroyo, J. M. Perez-Mato, D. Orobengoa, E. Tasci, G. de la Flor, and A. Kirov, Bulgarian Chemical Communications 43, 183 (2011).

51 E. S. Tasci, G. de La Flor, D. Orobengoa, C. Capillas, J. M. Perez-Mato, and M. I. Aroyo, in European Physical Journal Web of Conferences, European Physical Journal Web of Conferences, Vol. 22 (2012) p. 9.

${ }^{52}$ K. Momma and F. Izumi, Journal of Applied Crystallography 41, 653 (2008).

53 N. Marzari, A. A. Mostofi, J. R. Yates, I. Souza, and D. Vanderbilt, Rev. Mod. Phys. 84, 1419 (2012).

54 A. A. Mostofi, J. R. Yates, Y.-S. Lee, I. Souza, D. Vanderbilt, and N. Marzari, Computer Physics Communications 178, 685 (2008).

${ }^{55}$ H. Akamatsu, K. Fujita, H. Hayashi, T. Kawamoto, Y. Kumagai, Y. Zong, K. Iwata, F. Oba, I. Tanaka, and K. Tanaka, Inorganic Chemistry 51, 4560 (2012).

${ }^{56}$ H. Akamatsu, Y. Kumagai, F. Oba, K. Fujita, K. Tanaka, and I. Tanaka, Advanced Functional Materials 23, 1864 (2013).

57 V. Goian, S. Kamba, O. Pacherová, J. Drahokoupil, L. Palatinus, M. Dušek, J. Rohlíček, M. Savinov, F. Laufek, W. Schranz, A. Fuith, M. Kachlik, K. Maca, A. Shkabko, L. Sagarna, A. Weidenkaff, and A. A. Belik, Phys. Rev. B 86, 054112 (2012).

${ }^{58}$ It is pleasing that the original DFT paper determined a value of $U$ similar to this by comparing the calculated magnetic exchange parameters to experiment, and then predicted a $\omega_{S M}$ frequency remarkably close to the experiment.

${ }^{59}$ W. Cochran and R. A. Cowley, Journal of Physics and Chemistry of Solids 23, 447 (1962).
${ }^{60}$ I. B. Bersuker and B. G. Vekhter, Ferroelectrics 19, 137 (1978).

61 R. E. Cohen, Nature 358, 136 (1992).

62 K. Rabe, C. Ahn, and J. Triscone, Physics of Ferroelectrics: A Modern Perspective, Topics in applied physics (Springer-Verlag Berlin/Heidelberg, 2007).

${ }^{63}$ I. B. Bersuker, Phys. Rev. Lett. 108, 137202 (2012).

64 All of the Wannier functions presented in this study are calculated using an energy range that covers only the Eu $\mathrm{f}$ bands. No unentanglement is required since these bands are well separated in energy from others.

65 R. Ranjan, H. S. Nabi, and R. Pentcheva, Journal of Applied Physics 105, 053905 (2009).

66 While the exact quantitative value of $\sigma_{T i}$ depends on the details of the procedure used to calculate the site projected DOS, such as the radius of the spheres used; the two trends that we report are robust against changes in the sphere size.

67 D. J. Singh, M. Ghita, M. Fornari, and S. V. Halilov, Ferroelectrics 338, 1489+ (2006).

68 D. Khomskii, Journal of Magnetism and Magnetic Materials 306, 1 (2006).

69 N. Hill, Journal of Phsical Chemistry B 104, 6694 (2000).

70 Note that DFT with the LDA or GGA approximations is essentially a mean field theory $\stackrel{46}{4}$ and as a result such correlated processes are not included in it. However, the requirement that the Kohn-Sham states (and the corresponding Wannier states) are orthonormal essentially leads to the same result that if the Eu spins are antiparallel the $f$ electrons can delocalize to the $d$ states of the Ti ion more.

71 J. Haeni, P. Irvin, W. Chang, R. Uecker, P. Reiche, Y. Li, S. Choudhury, W. Tian, M. Hawley, B. Craigo, et al., Nature 430, 758 (2004).

72 S. Bhattacharjee, E. Bousquet, and P. Ghosez, Phys. Rev. Lett. 102, 117602 (2009).

73 Note, however, that the data obtained from the calculations in the AFM state (red) have a slightly larger slope than the one obtained from calculations in the FM state (blue). This indicates that while there are other contributions to spin-phonon coupling apart from the mechanism discussed in this study, they are relatively small.

74 T. Kolodiazhnyi, K. Fujita, L. Wang, Y. Zong, K. Tanaka, Y. Sakka, and E. Takayama-Muromachi, Applied Physics Letters 96 (2010).

75 N. Sai and D. Vanderbilt, Phys. Rev. B 62, 13942 (2000).

76 P. M. Woodward, Acta Crystallographica Section B 53, 44 (1997)

77 Note that the octahedral rotation angles obtained within DFT are roughly 3-4 degrees larger than the experimental value for both EuTiO3 and SrTiO3. This overestimation with respect to experiment within DFT is well-known to occur (see Ref. 75] and [79). Recent high-resolution powder diffraction data shows that in EuTiO3, the local rotation angle is much larger than the average one. ${ }^{36}$ The local value, which is about 8 degrees, agrees well with DFT.33.

78 A. Stroppa, M. Marsman, G. Kresse, and S. Picozzi, New Journal of Physics 12, 093026 (2010).

79 R. Wahl, D. Vogtenhuber, and G. Kresse, Phys. Rev. B 78, $104116(2008)$ 